\title{
Addressing physical health in mental illness: the urgent need to translate evidence-based interventions into routine clinical practice
}

\author{
B. O'Donoghue $\mathrm{O}^{1,2,3 * *}$ (i) \\ ${ }^{1}$ Orygen, Parkville, VIC, Australia \\ ${ }^{2}$ Centre for Youth Mental Health, University of Melbourne, Parkville, VIC, Australia \\ ${ }^{3}$ Department of Psychiatry, St Vincents University Hospital, Dublin South East Mental Health Services, Dublin, Ireland
}

\begin{abstract}
People affected by severe mental health disorders have a greatly reduced life expectancy compared to their non-affected peers. Cardiovascular disease is the main contributor to this early mortality, caused by higher rates of smoking, physical inactivity, unhealthy diet, sleep disturbance, excessive alcohol use or substance abuse and medication side effects. Therefore, we need to take a preventative approach and translate effective interventions for physical health into routine clinical practice. These interventions should be delivered across all stages of mental health disorders and could also have the added benefit of leading to improvements in mental health. Furthermore, we need to advocate to ensure that people affected by severe mental health disorders receive the appropriate medical assessments and treatments when indicated. This themed issue highlights that physical health is now an urgent priority for funding and development in mental health services. The widespread implementation of evidence-based interventions into routine clinical practice is an essential need for consideration by clinicians and policymakers.
\end{abstract}

Received 01 November 2020; Revised 25 January 2021; Accepted 29 January 2021

Key words: Physical health, Mortality, Cardiovascular disease, Mental health disorders.

\section{Introduction}

Over the last decade, there has been a paradigm shift in mental health services addressing the physical health of people affected by severe mental disorders. This response is appropriate as there is a wealth of research, amounting to nearly a 100 systematic review or metaanalyses, demonstrating that individuals affected by severe and enduring mental health disorders have more physical health comorbidities and a significantly reduced life expectancy compared to their peers (Firth et al. 2019). Early studies revealed that it was those with a diagnosis of schizophrenia who died approximately 20 years younger (Hjorthøj et al. 2017). However, we now know that this earlier mortality is associated with all of the severe and enduring mental health disorders, including depression and personality disorders (Walker et al. 2015). Tragically, this mortality gap is widening and has been described as 'at best a failure to act on evidence and at worse a form of lethal discrimination' (Thornicroft, 2013). Of late, there has been a shift in practice with mental health services beginning to assume some responsibility for addressing or advocating for the physical health of service users. However,

*Address for correspondence: Associate Professor B. O'Donoghue, Department of Psychiatry, Elm Mount Unit, St Vincent's University Hospital, Elm Park, Dublin 4, Ireland.

(Email: brian.odonoghue@orygen.org.au) while there is now sufficient knowledge that we need to address this issue, the most effective approach is not yet known. This themed issue on physical health aims to emphasise this critical issue and describe potential interventions to address the physical health of individuals affected by mental health disorders.

\section{Translation of evidence-based interventions into routine clinical practice}

This themed issue is timely, as, in the current circumstances of the COVID-19 pandemic, a significant proportion of the work of mental health services is being conducted via telehealth (Torous \& Wykes, 2020). While telehealth has its advantages, there may be some drawbacks in addressing physical health, as individuals are attending the clinic in person less frequently. Therefore, there is a risk that the progress made by mental health services in addressing physical health over the last decade could inadvertently be reversed. Furthermore, people are less active due to restrictions of the pandemic and practices that previously occurred at clinics, such as metabolic monitoring, are now more challenging. There are many facets to this challenge; the first is that we need to identify interventions that are effective for either preventing or reversing the poorer physical health associated with severe mental health disorders. At this point, there is sufficient evidence 
supporting specific interventions that are detailed in resources such as the recent Lancet Commission: 'A blueprint for protecting physical health in people with mental illness' (Firth et al. 2019) and also the 'Maudsley Practice guidelines for Physical Health Conditions in Psychiatry' (Taylor and Pillinger, 2020). The review on interventions to improve the physical health of people with a first episode of psychosis (FEP) by Fouhy et al. (2020) in this themed issue also highlights the limited, but effective interventions for this population (Fouhy et al. 2020).

Therefore, this issue does not just focus on determining what works, but rather how we could translate these evidence-based interventions into routine clinical practice - which is likely to be the greatest challenge in addressing physical health in mental health disorders. For example, at Orygen, a youth mental health service in Melbourne, we established an exercise physiology service that provided treatment to young people with a FEP, those with an at-risk mental state, severe personality disorders or depression (Pearce et al. 2020). The exercise physiologists provided individual and group programme sessions; however, we found that not all of the young people in need of the service were referred and there was suboptimal attendance and engagement for those who were referred (Pearce et al. 2020). Hence, an urgent focus is needed to translate what we know works from controlled, well-funded research settings into routine practice. Matthews et al. (2018) argue that this translation could be achieved by integrating specialised exercise practitioners into mental health multidisciplinary teams and providing training on physical activity and sedentary behaviour interventions to mental health clinicians (Matthews et al. 2018).

\section{Physical health interventions across the continuum of mental health disorders}

A staging model has been proposed in psychiatry in which the early stages are characterised by mild mental health symptoms to the more severe, relapsing disorders in the later stages (McGorry et al. 2006). It is proposed that treatments would differ at each point along this continuum, with interventions with lower risk-benefit ratios being provided in the early stages and more complex medical therapies in the later stages (Hickie et al. 2013). Physical health interventions for people with mental health disorders map neatly onto this proposed staging model, as physical health interventions could have different roles across this continuum. In the early stages, a focus on modifiable risk factors could derive multiple benefits. First, interventions focusing early on smoking, physical inactivity, unhealthy diet, sleep disturbance, and excessive alcohol use or substance abuse could lead to a positive contribution to the long-term physical health of the individual. These interventions could also have a preventative effect, as all of the above-mentioned lifestyle factors can contribute to the onset of mental health disorders (Firth et al. 2020). Therefore, it is possible that if we intervene early with lifestyle interventions, then progression to a further stage on the continuum could be prevented. In the later parts of the staging model, while preventative measures are still necessary, more assertive and targeted approaches are required. An example of this is in the management of treatment-resistant schizophrenia, for which clozapine is the most effective treatment (Leucht et al. 2013) but can have severe adverse effects on physical health (Tek et al. 2016). It is likely that lifestyle interventions alone would not be sufficient to counteract these adverse effects, and therefore, early use of interventions such as metformin are indicated (Siskind et al. 2016). Targeting these lifestyle factors in those who are prescribed clozapine could derive multiple benefits, for example, employing strategies to reduce the prevalence of smoking (Siskind et al. 2020), could result in achieving a therapeutic response with lower doses (Wagner et al. 2020), which in turn could lead to less metabolic side effects. Additionally, addressing obstructive sleep apnea, which is highly prevalent in this population, as demonstrated in this issue (Myles et al. 2020), could potentially lead to better mental health and physical outcomes.

Effective reform will require attention to all stages of this continuum, with services such as the physical health monitoring service for people prescribed longacting injectable medication described in this issue (Gill et al. 2016; Lydon et al. 2020). However, it needs to be ensured that there are effective interventions in place for people identified in the screening process. To achieve this holistic care, a suite of physical health interventions would need to be available, including dietetics, physical activity programmes to reduce sedentary behaviour, tobacco smoking cessation and substance use interventions. Addressing just one factor in isolation is unlikely to have a substantial impact. For example, while there would be significant health benefits by increasing physical activity and improving diet, it would be unlikely to achieve a substantial change in life expectancy if the individual continued to smoke tobacco (Tam et al. 2016). Hence, this calls for multiple, additional, resource-intensive interventions in mental health services that are already under-resourced. This leads to the next dilemma, who will provide these services?

\section{Advocacy and the interface between psychiatry and other medical specialities}

With the charge to address the physical health of people affected by mental health disorders, there has been less 
debate about who should deliver these services. Physical health is a broad term, and in addition to cardiovascular, respiratory and endocrine disorders, individuals with severe and enduring mental health disorders also have poorer sexual and reproductive health (Adan Sanchez et al. 2019) and dental health (Kisely, 2016). There is a limit to what is feasible and appropriate to be addressed by the mental health services based on resources, funding and expertise. Mental health services already take a collaborative approach to the care of people affected by mental health disorders by involving the affected individual, their caregivers, general practitioners and other health professionals. The same process should be taken in addressing physical health. A minimum standard could be determined by governing bodies and incorporated into the training of mental healthcare professionals. For anything beyond this, mental health services could negotiate with other health providers, such as general practitioners. The move towards establishing primary care centres in Ireland (Mental Health Reform, 2013), in which mental health and primary care are co-located, would make this a seamless process for the service user and their caregivers. The review by Fogarty et al. (2020) examines this issue and details the physical health issues of people affected by mental health disorders presenting to primary care (Fogarty et al. 2020).

Advocacy is another required role for the mental health services to undertake in addressing the physical health of people affected by mental health disorders. People with severe mental health disorders are less likely to receive the indicated investigations and interventions and this is referred to as 'diagnostic overshadowing', in which there is a misattribution of physical symptoms to mental illness. It can occur most often in Emergency Departments, particularly those that are crowded and busy or when the patient presents with challenging behaviour (Shefer et al. 2014). However, it also occurs in medical and surgical specialities, for example, in Western Australia people with severe mental health disorders and ischemic heart disease were less likely to undergo life-saving revascularisation procedures compared to those without mental health disorders (Lawrence et al. 2013). It is not surprising then that the mortality attributable to ischaemic heart disease had reduced in the general population in Western Australia, but this was not the case for people with severe mental health disorders (Lawrence et al. 2013). Diagnostic overshadowing is less common in Emergency Departments that have liaison psychiatry services (Shefer et al. 2014), indicating that advocacy from mental health professionals is effective in addressing this practice. However, this stigma and discrimination against people with mental health disorders need to be addressed at a systemic level. Considering the high prevalence of mental health disorders in the general population and their high rates of physical health comorbidities, knowledge of mental health disorders should be a component of all medical and surgical specialities training. For some of these specialties, the only exposure to psychiatry will have been a relatively brief placement in medical training.

\section{Mandating physical health monitoring}

There are some intriguing puzzles relating to physical health. The early mortality outcomes are superior for individuals treated with clozapine compared to individuals treated with other antipsychotic medication (Tiihonen et al. 2009). This is hard to fathom, considering that clozapine is associated with the greatest risk for weight gain (Leucht et al. 2013), diabetes in younger people (Lund et al. 2001), myocarditis (Bellissima et al. 2018) and blood dyscrasia (Latif et al. 2011). Yet, the finding of superior morbidity and mortality has been replicated many times (Cho et al. 2019; Wimberley et al. 2017). One of the leading theories is that physical health monitoring is mandatory for the ongoing prescribing of the medication, thereby ensuring that individuals taking clozapine attend the mental health service monthly, thereby facilitating regular contact with their treating team. Pharmacists cannot dispense the prescribed clozapine until a neutrophil count is known, and this mechanism ensures $100 \%$ compliance. This procedure is accepted as being part of the process of prescribing clozapine. However, it needs to be asked why this process is only applied to a rare side effect (albeit potentially fatal) but not to the more common side effects, specifically metabolic side effects that strongly contribute to the early mortality in this population. Therefore, a drastic but potentially effective intervention could be to mandate regular anthropometric measurements, fasting glucose and lipids as a requirement for the ongoing prescription of all antipsychotic medication. This would ensure compliance with physical health screening and assist in the provision of interventions when indicated.

\section{Ambitious and achievable targets}

We need ambitious but achievable targets to bring about change. For example, the Healthy Active Lives (HeAL) declaration sets out a 5-year target for young people experiencing a FEP (International Physical Health in Youth (IPHYS) Working Group, 2013). It stipulates that young people with an FEP should have the same life expectancy as their non-affected peers, in that clinically significant weight gain should occur in less than $25 \%$ and that less than $30 \%$ smoke tobacco. It also 
sets out the target that more than half of people with an FEP engage in levels of physical activity appropriate for their age. These targets are ambitious and should be commended; however, they also need tiriso be supported by resources and training. To adequately manage the physical health of people with severe mental health disorders, clinicians will need to adapt their regular practice, which will require education and training. Many governing bodies have developed training modules on addressing physical health that have been incorporated into the training requirements for psychiatrists in some jurisdictions, such as Australia with the Royal Australian and New Zealand College of Psychiatrist (Lambert et al. 2017). The recent report on the 'Physical Health of People with severe mental illness' by the Mental Health Commission highlighted that specialist training in mental health should not be at the cost of treating the 'whole' patient (Finnerty, 2019).

\section{Conclusion}

Despite early mortality in severe mental health disorders being established for decades, the gap in life expectancy between those with and without severe and enduring mental health disorders continues to widen (Lawrence et al. 2013). The vast majority of this early mortality is attributable to preventable causes such as cardiovascular disease. Therefore, we need to take a preventative approach and translate effective interventions for physical health into routine clinical practice. Furthermore, we need to advocate to ensure that people affected by mental health disorders receive the appropriate medical assessments and treatments when indicated. Concurrently, we need to develop more effective, safe and acceptable interventions that can be delivered across the continuum of mental health disorders. This themed issue highlights that physical health is now an urgent priority for funding and development in mental health services and the widespread implementation of evidence-based interventions in routine clinical practice is an essential need for consideration by clinicians and policymakers.

\section{Financial support}

This article received no specific grant from any funding agency, commercial or not-for-profit sectors (or declare financial support if appropriate). Associate Professor Brian O'Donoghue is supported by the National Health and Medical Research Council (NHMRC) research fellowship.

\section{Conflict of interest}

The author has no conflict of interest to disclose.

\section{Ethical standards}

The author asserts that all procedures contributing to this work comply with the ethical standards of the relevant national and institutional committee on human experimentation with the Helsinki Declaration of 1975, as revised in 2008. The author asserts that ethical approval was not required for the publication of this manuscript.

\section{References}

Adan Sanchez AY, Mcmillan E, Bhaduri A, Pehlivan N, Monson K, Badcock P, Thompson K, Killackey E, Chanen A, O'donoghue B (2019). High-risk sexual behaviour in young people with mental health disorders. Early Intervention in Psychiatry 13, 867-873.

Bellissima BL, Tingle MD, Cicović A, Alawami M, Kenedi C (2018). A systematic review of clozapineinduced myocarditis. International Journal of Cardiology 259, 122-129.

Cho J, Hayes RD, Jewell A, Kadra G, Shetty H, Maccabe JH, Downs J (2019) Clozapine and all-cause mortality in treatment-resistant schizophrenia: a historical cohort study. Acta Psychiatrica Scandinavica 139, 237-247.

Finnerty S (2019). Physical Health of People with Severe Mental Illness. Mental Health Commission: Ireland.

Firth J, Siddiqi N, Koyanagi A, Siskind D, Rosenbaum S, Galletly C, et al. (2019). The Lancet psychiatry commission: a blueprint for protecting physical health in people with mental illness. Lancet Psychiatry 6, 675-712.

Firth J, Solmi M, Wootton RE, Vancampfort D, Schuch FB, Hoare E, et al. (2020). A meta-review of "lifestyle psychiatry": the role of exercise, smoking, diet and sleep in the prevention and treatment of mental disorders. World Psychiatry 19, 360-380.

Fogarty F, Mccombe G, Brown K, Van Amelsvoort T, Clarke M, Cullen W (2020). Physical health among patients with common mental health disorders in primary care in Europe: a scoping review. Irish Journal of Psychological Medicine, 1-17. Published online ahead of print.

Fouhy F, Cullen W, O'connor K (2020). Physical health interventions for patients who have experienced a first episode of psychosis: a narrative review. Irish Journal of Psychological Medicine, 1-14. Published online ahead of print.

Gill M, Mckenna K, Mccauley M, Gulzar M (2016). Establishing a physical health monitoring service for patients on depot antipsychotic medication. Irish Journal of Psychological Medicine, 1-7. Published online ahead of print.

Hickie IB, Scott J, Mcgorry PD (2013). Clinical staging for mental disorders: a new development in diagnostic practice in mental health. Medical Journal of Australia 198, 461-462. 
Hjorthøj C, Stürup AE, Mcgrath JJ, Nordentoft M (2017). Years of potential life lost and life expectancy in schizophrenia: a systematic review and meta-analysis. Lancet Psychiatry 4, 295-301.

International Physical Health in Youth (IPHYS) Working Group (2013). Healthy Active Lives (HeAL) Consensus Statement (https://www.iphys.org.au/). Accessed 25 January 2021.

Kisely S (2016). No mental health without oral health. Canadian Journal of Psychiatry 61, 277-282.

Lambert TJ, Reavley NJ, Jorm AF, Oakley Browne MA (2017). Royal Australian and New Zealand college of psychiatrists expert consensus statement for the treatment, management and monitoring of the physical health of people with an enduring psychotic illness. Australian and New Zealand Journal of Psychiatry 51, 322-337.

Latif Z, Jabbar F, Kelly BD (2011). Clozapine and blood dyscrasia. The Psychiatrist 35, 27-29.

Lawrence D, Hancock KJ, Kisely S (2013). The gap in life expectancy from preventable physical illness in psychiatric patients in Western Australia: retrospective analysis of population based registers. BMJ 346, f2539.

Leucht S, Cipriani A, Spineli L, Mavridis D, Orey D, Richter F, Samara M, Barbui C, Engel RR, Geddes JR, Kissling W, Stapf MP, Lassig B, Salanti G, Davis JM (2013). Comparative efficacy and tolerability of 15 antipsychotic drugs in schizophrenia: a multipletreatments meta-analysis. Lancet 382, 951-962.

Lund BC, Perry PJ, Brooks JM, Arndt S (2001). Clozapine use in patients with schizophrenia and the risk of diabetes, hyperlipidemia, and hypertension: a claims-based approach. Archives of General Psychiatry 58, 1172-1176.

Lydon A, Vallely J, Tummon A, Maher S, Sabri S, Mcloughlin J, Liew A, Mcdonald C, Hallahan B (2020). Routine screening and rates of metabolic syndrome in patients treated with clozapine and long-acting injectable antipsychotic medications: a cross-sectional study. Irish Journal of Psychological Medicine, 1-9. Published online ahead of print.

Matthews E, Cowman M, Denieffe S (2018). The next step: a strategic focus on physical activity and sedentary behaviour in Irish mental health care. Irish Journal of Psychological Medicine, 1-3. Published online ahead of print.

Mcgorry PD, Hickie IB, Yung AR, Pantelis C, Jackson HJ (2006). Clinical staging of psychiatric disorders: a heuristic framework for choosing earlier, safer and more effective interventions. Australian and New Zealand Journal of Psychiatry 40, 616-622.

Mental Health Reform (2013). Mental Health in Primary Care in Ireland: A briefing paper (www.mentalhealthreform.ie). Accessed 25 January 2021.

Myles H, Myles N, Vincent AD, Wittert G, Adams R, Chandratilleke M, Liu D, Mercer J, Vakulin A, Chai-Coetzer CL, Galletly C (2020). Pilot cohort study of obstructive sleep apnoea in community-dwelling people with schizophrenia. Irish Journal of Psychological Medicine, 1-7. Published online ahead of print.

Pearce M, Foote L, Brown E, O'donoghue B (2020).

Evaluation of an exercise physiology service in a youth mental health service. Irish Journal of Psychological Medicine, 1-6. Published online ahead of print.

Shefer G, Henderson C, Howard LM, Murray J, Thornicroft G (2014). Diagnostic overshadowing and other challenges involved in the diagnostic process of patients with mental illness who present in emergency departments with physical symptoms-a qualitative study. PloS One 9, e111682-e111682.

Siskind DJ, Leung J, Russell AW, Wysoczanski D, Kisely S (2016). Metformin for clozapine associated obesity: a systematic review and meta-analysis. PloS One 11, e0156208-e0156208.

Siskind DJ, Wu BT, Wong TT, Firth J, Kisely S (2020). Pharmacological interventions for smoking cessation among people with schizophrenia spectrum disorders: a systematic review, meta-analysis, and network metaanalysis. Lancet Psychiatry 7, 762-774.

Tam J, Warner KE, Meza R (2016). Smoking and the reduced life expectancy of individuals with serious mental illness. American Journal of Preventative Medicine 51, 958-966.

Taylor DGF, Pillinger T (2020). The Maudsley Practice Guidelines for Physical Health Conditions in Psychiatry. John Wiley \& Sons Inc: West Sussex, UK.

Tek C, Kucukgoncu S, Guloksuz S, Woods SW, Srihari VH, Annamalai A (2016). Antipsychotic-induced weight gain in first-episode psychosis patients: a meta-analysis of differential effects of antipsychotic medications. Early Intervention in Psychiatry 10, 193-202.

Thornicroft G (2013). Premature death among people with mental illness. BMJ 346, f2969.

Tiihonen J, Lonnqvist J, Wahlbeck K, Klaukka T, Niskanen L, Tanskanen A, Haukka J (2009). 11-year follow-up of mortality in patients with schizophrenia: a population-based cohort study (FIN11 study). Lancet 374, 620-627.

Torous J, Wykes T (2020). Opportunities from the coronavirus disease 2019 pandemic for transforming psychiatric care with telehealth. JAMA Psychiatry 77, 1205-1206.

Wagner E, Mcmahon L, Falkai P, Hasan A, Siskind D (2020). Impact of smoking behavior on clozapine blood levels - a systematic review and meta-analysis. Acta Psychiatrica Scandinavica 142, 456-466.

Walker ER, Mcgee RE, Druss BG (2015). Mortality in mental disorders and global disease burden implications: a systematic review and meta-analysis. JAMA Psychiatry 72, 334-341.

Wimberley T, Maccabe JH, Laursen TM, Sørensen HJ, Astrup A, Horsdal HT, Gasse C, Stovring H (2017). Mortality and self-harm in association with clozapine in treatment-resistant schizophrenia. American Journal of Psychiatry 174, 990-998. 